\title{
A Comprehensive Histopathological Study of Skin Appendageal Tumors
}

\author{
Dr. Chitrakala Sugumar ${ }^{1}$, Dr.Gayathri.G ${ }^{2,}$ Dr. Bharathi Vidhya Jayanthi ${ }^{3}$ \\ ${ }^{I}$ (Associate Professor, Department Of Pathology/ Thiruvannamalai Medical College, Thiruvannamalai, India) \\ ${ }_{2}^{2}$ (Assistant Professor, Department Of Pathology/ Kilpauk Medical College, Chennai, India) \\ ${ }^{3}$ (Professor, Department Of Pathology/ Kilpauk Medical College Chennai, India)
}

\begin{abstract}
:
Background: The Skin Appendageal tumors are a heterogenous group of neoplasms with distinct histological features. The aim of this study is to determine the incidence, age, sex, site, histopathological features and the pattern of differentiation of the appendageal tumors analogous to hair follicles, sebaceous, eccrine and apocrine glands.

Materials and methods : This is a retrospective study done in a tertiary care hospital in South India that includes 52 cases of skin appendageal tumors reported at the Department of Pathology, Government Kilpauk Medical College, Chennai, over a period of three years.

Results : During the three year period of this study, a total of 1532 skin biopsies were reported, of which 52 cases were skin appendageal tumors(3.4\%).Benign tumors were 50(96.2\%) and malignant were 2(3.8\%).The mean age of presentation was 38.2 years. The male:female ratio was 1:1.6. The tumors were predominant in females $32(61.5 \%)$ in comparison to males 20 cases(38.5\%). The eccrine tumors constituted $21(40.4 \%)$ and thus were the most common, followed by tumors of hair follicular differentiation 17(32.7\%). The commonest site was face in 20 cases(38.4\%), followed by scalp in 15 cases(28.8\%). The common clinical presentation was nodular and papular lesions. The commonest lesion was pilomatrixoma 13(25\%), followed by nodular hidradenoma 9 cases $(17.3 \%)$

Conclusion : The incidence of skin appendageal tumors in our study is $3.4 \%$ and thus they are relatively uncommon. Their clinical diagnosis is difficult as many appendageal tumors have similar clinical presentation and hence histopathology is mandatory and gold standard for diagnosis. Benign skin appendageal tumors are more common than the malignant tumors, which are seen in elderly patients.
\end{abstract}

Keywords: Skin, Appendageal, Benign, malignant, histopathology

\section{Introduction}

Skin appendageal tumors encompass a wide variety of tumors with distinct histological features[1-3]. They are classified into four groups according to their appendageal differentiation as follicular, sebaceous, eccrine and apocrine[1-4]. These tumors generally are benign but malignant tumors are also reported[ 2,16] Most of the appendageal tumors are asymptomatic or present as papules and nodules[5]. The most common site is the face[2,17]. In our present study a comprehensive histopathological analysis of the skin appendageal tumors is done and their clinicopathological correlation with respect to age, sex, site and the appendageal differentiation was made.

\section{Materials And Methods}

This is a retrospective study done in a tertiary care hospital in south India that includes 52 cases of skin appendageal tumors reported at the Department of Pathology, Government Kilpauk Medical College, Chennai, over a period of three years between January2013- January2016. The clinicopathological data were collected from office records and the department of dermatology during the given period .The histo pathological examination was done on formalin fixed tissues. Haematoxylin and eosin stained sections were examined. Special stains like PAS and Van Gieson was done when needed. The tumors were classified as follicular, sebaceous, eccrine and apocrine[6]. Clinicopathological correlation was made.

\section{Results}

During the three year period, a total number of 1532 skin biopsies were reported, of which ,the skin appendageal tumors was 52(3.4\% of skin cases). Benign tumors were 50(96.2\%) and malignant were 2(3.8\%). The mean age of presentation was 38.2 years. The tumors were predominant in females $32(61.5 \%)$ in comparison to males 20 cases(38.5\%).The male : female ratio is 1:1.6 as shown in Table1, "Fig1." 


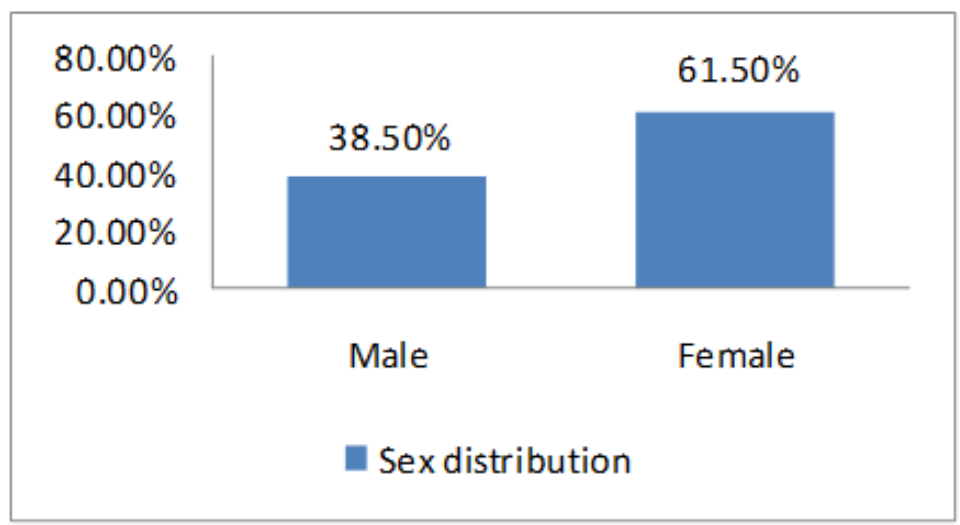

Fig1: Sex distribution of appendageal tumors

The eccrine tumors constituted 21(40.4\%) and thus were the most common, followed by tumors of hair follicular differentiation $17(32.7 \%)$. Tumors with sebaceous differentiation were $6(11.5 \%)$ and apocrine were $8(15.4 \%)$."Fig2."

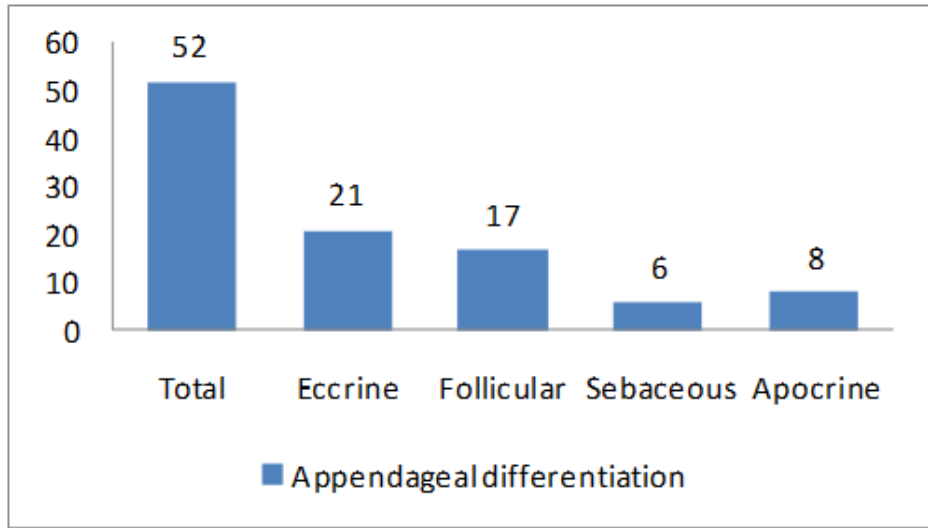

Fig2: Types of Appendageal tumors

Face was the commonest site 20(38.4\%), followed by scalp in 15 cases(28.8\%), extremities 13 cases $(25 \%)$ and trunk 4(7.6\%). "Fig3."

\section{Appendageal tumor site distribution}

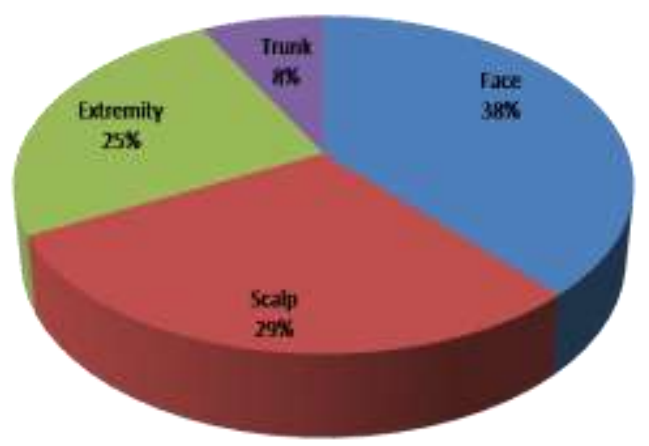

Fig3: Site distribution of Appendageal tumors

The common clinical presentation was nodular and papular lesions. The commonest lesion was pilomatrixoma $13(25 \%)$, followed by nodular hidradenoma 9 cases $(17.3 \%)$. The rare cases reported were eccrine spiradenoma, trichoadenoma, apocrine hidrocystoma. The rare and malignant cases reported were malignant nodular hidradenoma and poro carcinoma. 
Table 1.Comprehensive analysis of skin Appendageal Tumors:

\begin{tabular}{|c|c|c|}
\hline \multirow[t]{2}{*}{ Behaviour } & Benign & $50[96.2 \%]$ \\
\hline & Malignant & $2(3.8 \%)$ \\
\hline \multirow[t]{3}{*}{ Sex } & Male & $20(38.5 \%)$ \\
\hline & Female & $32[61.5 \%)$ \\
\hline & Male: Female Ratio & $1: 1.6$ \\
\hline \multirow[t]{7}{*}{ Age } & 0-10yтs & $3(5.8 \%)$ \\
\hline & 11-20yrs & $6(11.5 \%)$ \\
\hline & 21-30yrs & $9(17.33)$ \\
\hline & $31-40 \mathrm{yrs}$ & $18[34.6 \%)$ \\
\hline & 41-50yrs & $5(9.68)$ \\
\hline & 5160 prs & $6(11.6 \%)$ \\
\hline & 61-70yrs & $5\left(9.6 x_{0}\right)$ \\
\hline \multirow[t]{4}{*}{ Site } & Scalp & $15(28.8 \%)$ \\
\hline & Face & $20(38.486)$ \\
\hline & Extremities & $13(25 \%)$ \\
\hline & Trunk & $4\left(7.6 x_{0}\right)$ \\
\hline \multirow[t]{4}{*}{ Appendageal differentiation } & Eocrine & $21(40.4 \%)$ \\
\hline & Hair follicular & $17(32.7 \%)$ \\
\hline & Sebaceous & $6(11.5 \%)$ \\
\hline & Apocrine & $8(15.4 \times)$ \\
\hline Commonest Tumor & Pilomatricoma & $13(25 \%)$ \\
\hline \multirow[t]{2}{*}{ Malignanttumors } & Malignant excrine poroma & $1(1.9 \%)$ \\
\hline & Malignant nodular Hidraadenoma & $1(1.9 \%)$ \\
\hline
\end{tabular}

\section{Discussion}

A total number of 1532 skin biopsies were reported, of which, the skin appendageal tumors were 52 (3.4\% of skin cases).Thus they are not common. The Skin appendageal tumors are a large heterogenous group of neoplasms with distinct histological features. They are classified into four groups analogous to their appendageal differentiation as follicular, sebaceous, eccrine and apocrine.[5-8] These tumors generally are benign, but malignant types also exist[9]. A total of 52 appendageal tumours were observed in the current study. Of which the eccrine tumors constituted 21 cases $(40.4 \%), 17(32.7 \%)$ were of hair follicular differentiation, $6(11.5 \%)$ were sebaceous gland tumors and $8(15.4 \%)$ were apocrine tumors.

\section{Tumors With Eccrine Differentiation.}

Eccrine tumors were the most common in our study. Most of them were benign

1.1. Nodular Hidradenoma: Nodular hidradenoma, also referred to as clear cell hidradenoma, clear cell myoepithelioma and eccrine acrospiroma, was the second commonest appendageal tumor observed in our study. 9 cases of nodular Hidradenoma and one case of malignant nodular hidradenoma was reported in the present study[10]. The nodular hidradenoma was observed in various sites, with predilection to scalp and commonly presenting in the age group of 30-40 years. The tumor was well circumscribed and appeared encapsulated. It was composed of multiple epithelial lobules located in the dermis and within the lobulated masses, tubular lumina and focal cystic spaces were seen. The tumor showed two types of cells, clear cells which are round and containing a very clear cytoplasm with a small dark nuclei and the polyhedral cells with round nucleus and basophilic cytoplasm. "Fig4."

1.2. Eccrine Poroma: Of the 4 cases of eccrine poroma observed in our study, 3 presented as firm nodules in the extremities and one in the chest. Histologically, the tumor showed downward extensions into the dermis as broad anastomosing bands of epithelial cells emanating from the epidermis. The border between epidermis and dermis was readily apparent because of the distinctive appearance of the tumor cells, which are smaller than the keratinocytes. The cells were uniform cuboidal appearance and connected by intercellular bridges. A ductal lumen was also present."Fig5" 
1.3. Eccrine Spiradenoma: 4 cases of eccrine spiradenoma were reported in the present study, and all the 4 were located in the extremities, 2 in the upper and 2 in the lower extremity[2]. They all presented as painful nodules, clinically[11]. The tumor consisted of well circumscribed lobules containing intertwining cords of epithelial cells, surrounded by capsule of compressed connective tissue. The cords showed two types of cells, small basophilic cells with dark nuclei at the periphery and large cells with pale nuclei in the center and surrounded by basement membrane like material. "Fig6."

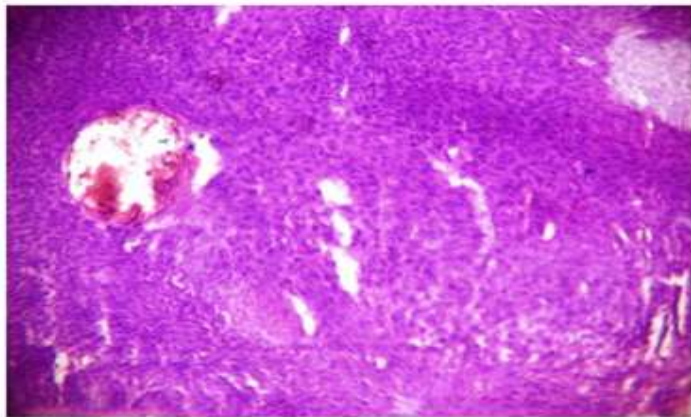

Fig.4: Nodular Hidradenoma

Tumor lobules showing clear \& polyhedral cells

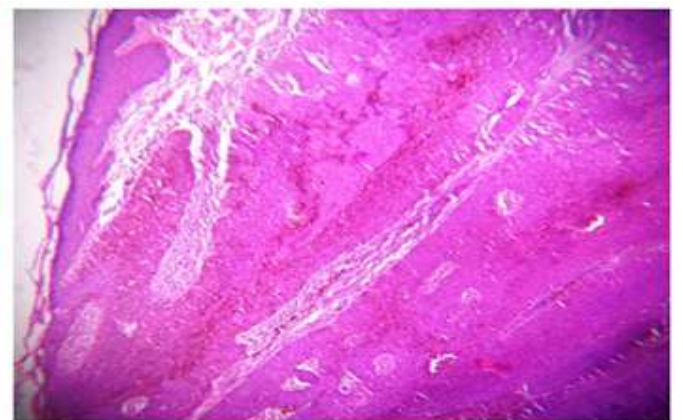

Fig.5: Eccrine Poroma Broad anastomosing bands from epidermis

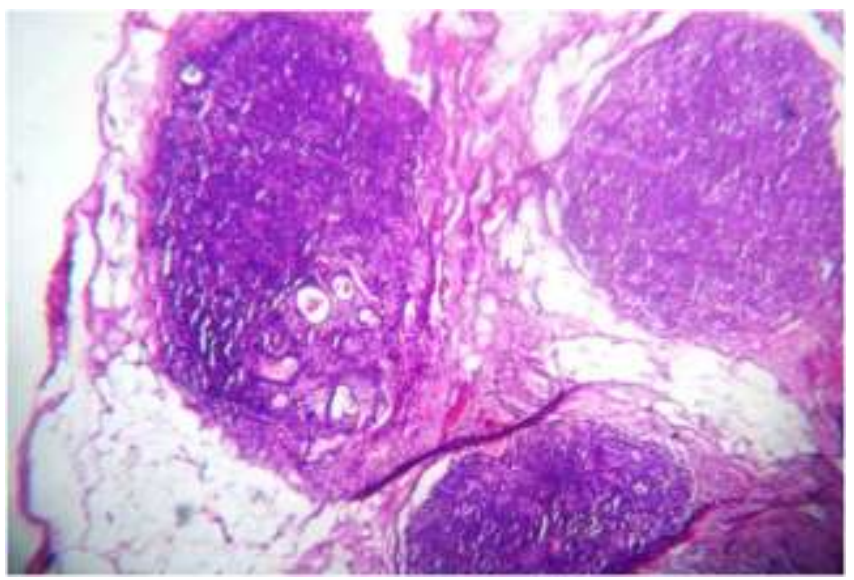

Fig.6: Eccrine Spiradenoma: Well circumscribed aggregates of tumor cells arranged in intertwining cords

1.4. Chondroid Syringoma: Mixed tumor of skin, the term introduced by Hirsch and Helwig in 1961, has been replaced by the term Chondroid Syringoma. A single case was observed in the study and it was a firm intradermal nodule in face.[12] Chondroid syringomas occur more commonly in head and neck. Similar observations were observed in our study. Histologically, tubular lumina with two layers of epithelial cells in a chondroid matrix is noted. "Fig7."

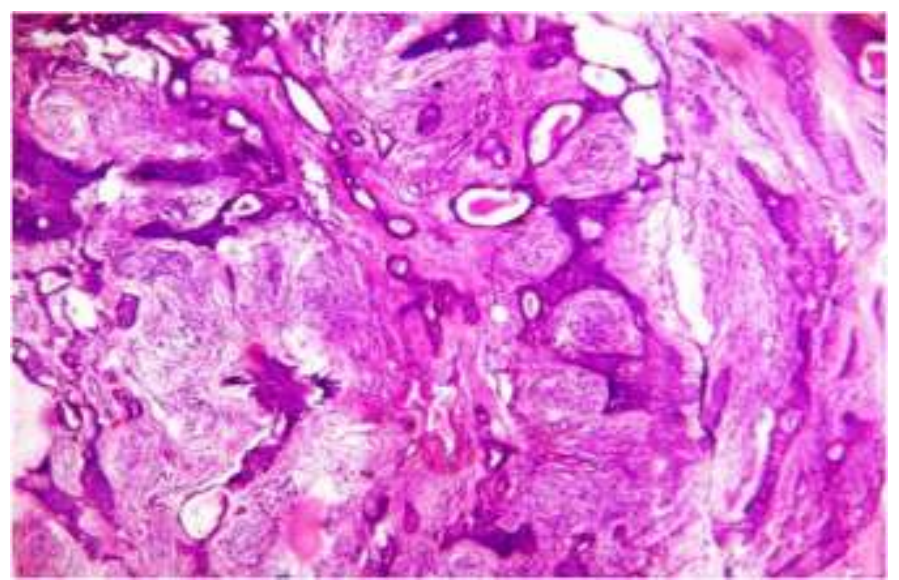

Fig.7: Chondroid syringoma: Tubular lumina lined by two layers of epithelial cells \& abundant chondro myxoid stroma 
1.5. SYRINGOMA: A single case of syringoma was reported in the present study. It was located in the lower eyelid. Histopathology revealed a fibrous stroma with numerous small ducts, the walls of which were lined by two rows of epithelial cells. Some ducts were lined by an eosinophilic cuticle, others had comma like tails. "Fig8."

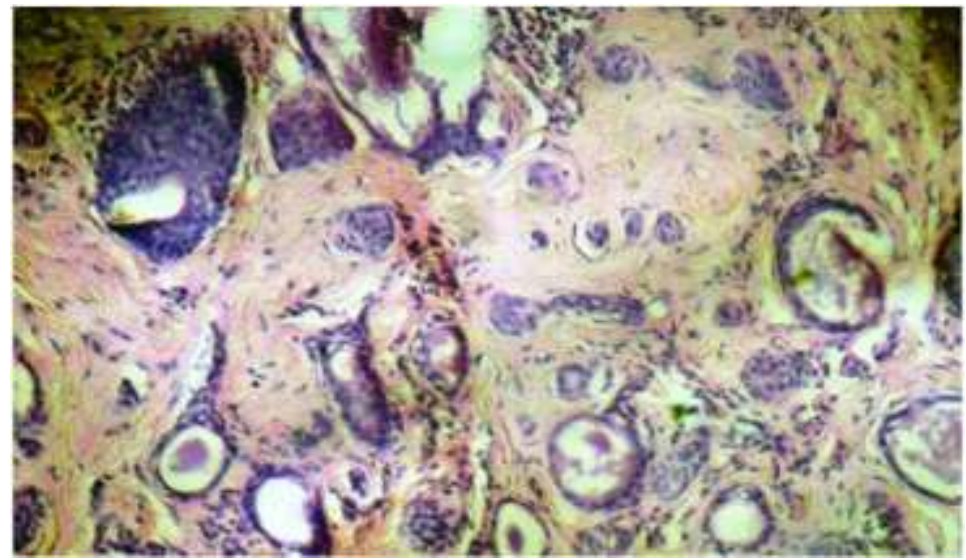

Fig.8: Syringoma: Ducts lined by two layers of epithelial cells, some showing comma like tails

1.6. Malignant Nodular Hidradenoma: One rare case of malignant nodular hidradenoma was observed in our study in a 70 year old male presenting as a nodular lesion in the scalp..These lesions tend to metastasise and may cause death. Histopathology showed malignant nodular hidradenoma as large asymmetrical tumor with invasion into surrounding tissues. The tumor was highly cellular, with cytological atypia and mitosis was also detected[10]. In some cases, nuclear anaplasia may be only slight to moderate and may be limited usually only to the clear cells.

1.7. Malignant Eccrine Poroma (Porocarcinoma): One rare case of malignant porocarcinoma was observed in the present study series. Though the tumor favors to occur in the extremities, in our case, the lesion was located in the scalp in a 65 year old female. Microscopy showed a malignant neoplasm arising from epidermis as large islands of tumor cells and extending into the dermis. The tumor was composed of nests of malignant cells exhibiting hyperchromatic pleomorphic nuclei, many with central necrosis. Some cell nests also showed squamous differentiation. "Fig9,10." The stroma was fibrotic and desmoplastic, interspersed with groups and nests of atypical cells, mitosis were also seen. [13]

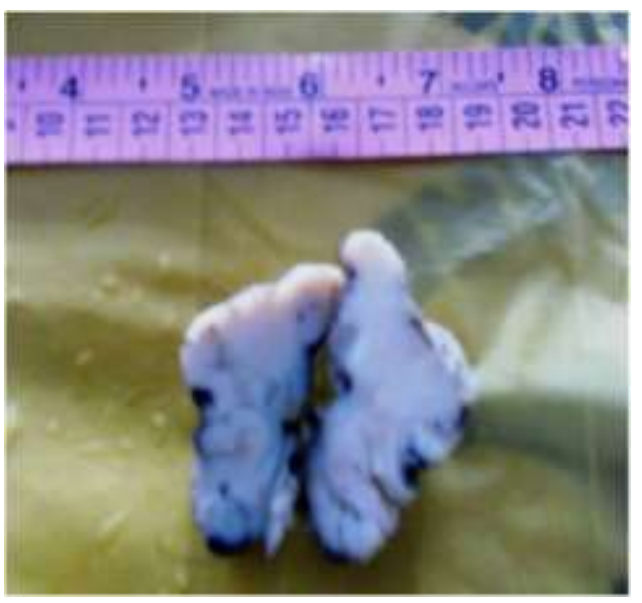

Fig.9: Poro carcinoma

C/S: Gray white firm nodular appearance

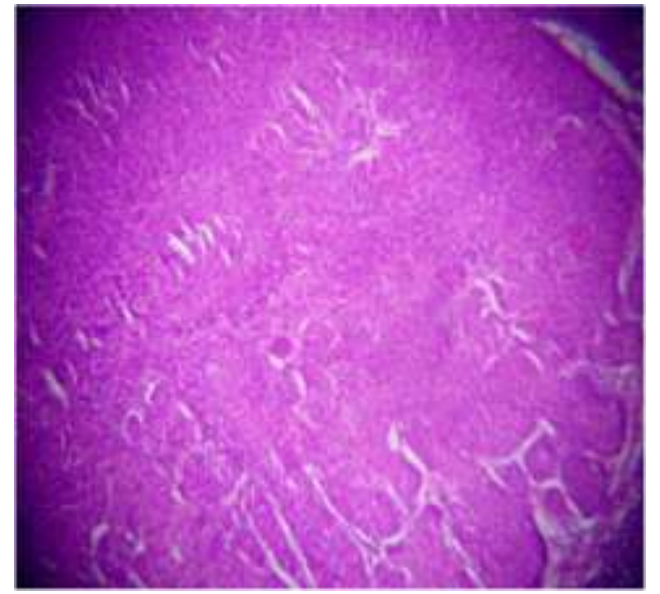

Fig.10: Poro carcinoma

Islands of tumor cells with nuclear atypia \& necrosis

\section{Tumors With Apocrine Differentiation}

2.1. Apocrine Hidrocystoma: One rare case of apocrine hidrocystoma was reported in the present study that occurred in the face, which is the usual site noted in various studies also[14]. Histopathological examination showed several large cystic spaces into which papillary projections extend. The inner surface of the cyst and the papillary projections were lined by two layers of cells, an inner row of columnar secretory cells showing 
decapitation secretions, indicative of apocrine secretion. Peripheral to the secretory cell layer are elongated myoepithelial cells"Fig11.".
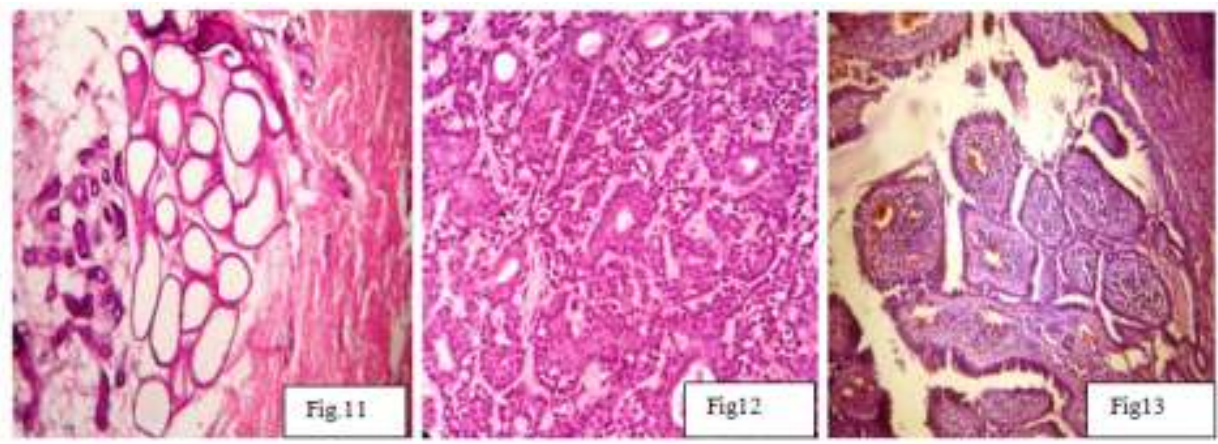

Fig.11: Apocrine hidrocystoma: Multiloculated cysts lined by inner columnar \& outer myoepithelial cells and showing decapitation secretions.

Fig.12: Cylindroma: Islands of epithelial cells arranged in jigsaw puzzle and surrounded by hyaline basement membrane.

Fig13. Syringocystadenoma papilliferum: Cystic invaginations with papillary projections lined by two layers of epithelial cells.

2.2. Hidradenoma Papilliferum: A single case of hidradenoma papilliferum was observed in this study. It usually occurs in the labia majora or in the perineal region in females. But in our study, it was a male patient in the gluteal region. Located in the dermis, it was a well circumscribed lesion, surrounded by a fibrous capsule with no connection to the overlying epidermis. There were scattered tubular cystic and branching spaces. Numerous papillary folds lined by double layer of cells, an inner layer of secretory cells and an outer layer of small cuboidal cells were seen projecting into the cystic spaces.

2.3. Cylindroma: Two cases of cylindroma were observed in the present study and both appeared as solitary lesions, in the scalp[14]. Cylindromas represent a tumor that may manifest both apocrine and eccrine differentiation, however the features of apocrine differentiation predominate. Cylindromas are composed of irregularly shaped islands of epithelial cells that fit together like pieces of jigsaw puzzle. The islands were surrounded by hyaline sheath, of variable thickness, like a cylinder.. Two cell types were seen, periphery showing small cells with dark staining nuclei often in a palisaded arrangement and the center of the islands showing large cells with light staining nuclei. "Fig12."

2.4. Syringocystadenoma Papilliferum: 4 cases of Syringocystadenoma papilliferum observed in the present study were commonly found in the scalp and face.[2,17] Histologically, epidermis showed cystic invaginations lined by keratinizing squamous cells in the upper part and papillary projections lined by two rows of epithelium in the lower part. The stroma was densely cellular with plasma cells. "Fig13."

\section{Tumors with Sebaceous Differentiation}

3.1. Nevus Sebaceous: 4 cases of nevus sebaceous were reported in our study and 3 were located in the face and 1 in the scalp, which are the common sites. Histologically it showed proliferations of sebaceous glands, papillomatous epidermis and ectopic apocrine glands deep in the dermis.

3.2. Sebaceous Adenoma: 2 cases of sebaceous adenoma were reported in the case series and both were located in the scalp, which is the common site observed in various studies. It is composed of incompletely differentiated sebaceous lobules that are irregular in size and shape. The lobules contain two types of cells, basaloid and the sebaceous, with the sebaceous cells predominating.

\section{Tumors with Hair Follicular Differentiation:}

Histopathologically $32.7 \%$ of tumors showed follicular differentiation, compared to $63.4 \%$ by Jayalakshmi et al [9].

4.1. Pilomatricoma (Calcifying Epithelioma Of Malherbe): Pilomatricoma are benign tumours with differentiation towards hair matrix.[15]. 13 cases of Pilomatricoma observed in the current study were found in face and upper extremities occurring more commonly in the age group of less than 20 years[16]. Histologically, 
the tumor was sharply demarcated and encapsulated. It was located in the lower dermis and composed of irregular shaped islands of two types of epithelial cells (basophilic cells and shadow cells) with calcification and foreign body reaction"Fig14.". Malignant transformation in Pilomatricoma was not observed in the present study.
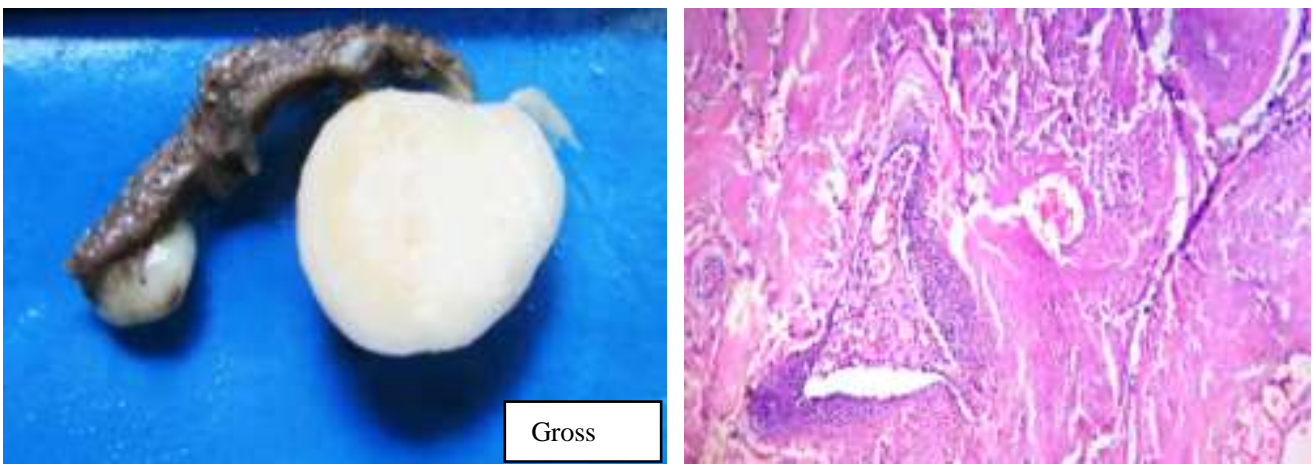

Fig.14: Pilomatricoma: Basaloid cells and shadow cells with trichilemmal keratinisation

4.2. Trichoepithelioma: One case of Trichoepithelioma was observed in the study. It presented as multiple lesions in the form of skin coloured papules mainly in the nose and nasolabial fold[17,18]. Histologically, horn cysts are characteristically seen that consists of a fully keratinized centre surrounded by basophilic cells that show peripheral palisading. "Fig15." Important histopathological differential diagnosis is Basal Cell Carcinoma and was differentiated by the absence of retraction artifact of stroma. Papillary mesenchymal bodies,amyloid deposits, mitosis and inflammatory granuloma are other features that distinguish trichoepithelioma from Basal cell Carcinoma.

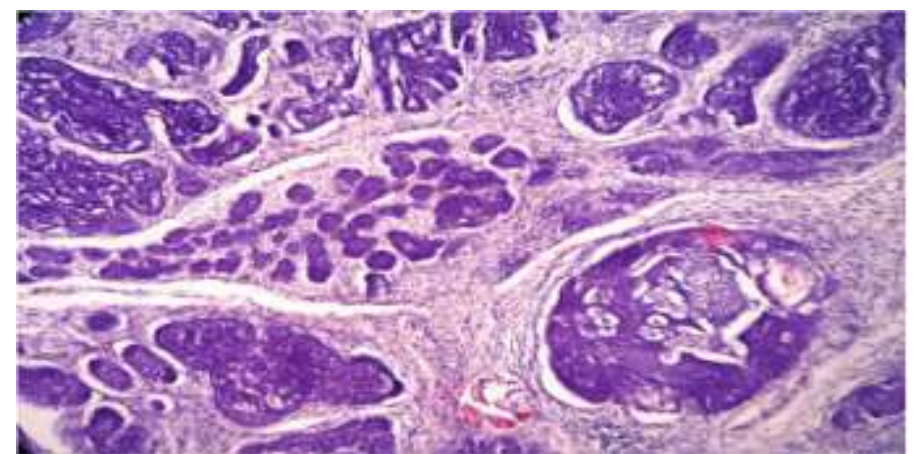

Fig15: Trichoepithelioma: Nests of basaloid cells, horn cysts \& papillary mesenchymal bodies

4.3. Trichofolliculoma: 1 case of trichofolliculoma was reported in our study series. The site was face..Histology showed the dermis consisting of large cystic spaces lined by squamous epithelium and radiating from this primary hair follicles, there were many well developed secondary hair follicles.

4.4. Proliferating Trichilemmal Tumor: One case of the proliferating trichilemmal cyst also referred to as proliferating trichilemmal tumor, was reported in the present study series. The site of occurrence was the scalp, in concordance with other studies[17]. Histologically, the tumor was composed of multiple lobules of squamous epithelium with peripheral palisading. The squamous epithelium characteristically showed abrupt trichilemmal keratinisation. "Fig16."

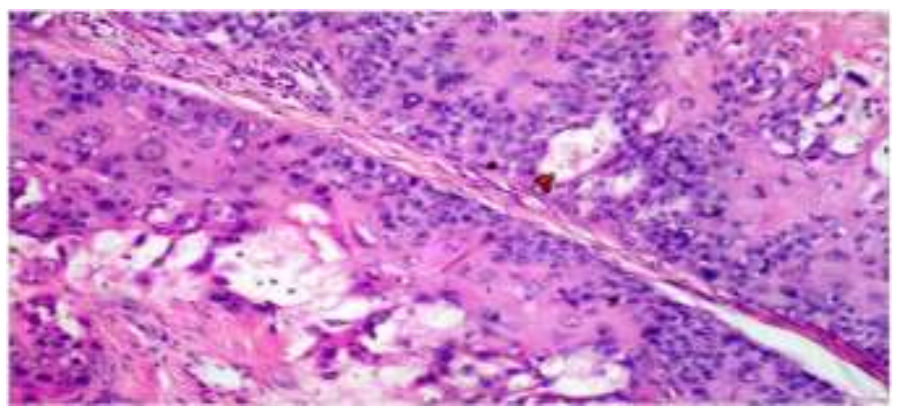

Fig16: Proliferating trichilemmal tumor: Squamous epithelium showing abrupt trichilemmal keratinisation 
4.5. Trichoadenoma: A rare solitary tumor first described in 1958, trichoadenoma usually occurs in the face, as in our study. Histopathology showed numerous horn cysts containing keratin distributed throughout the dermis, surrounded by eosinophilic cells"Fig17.".

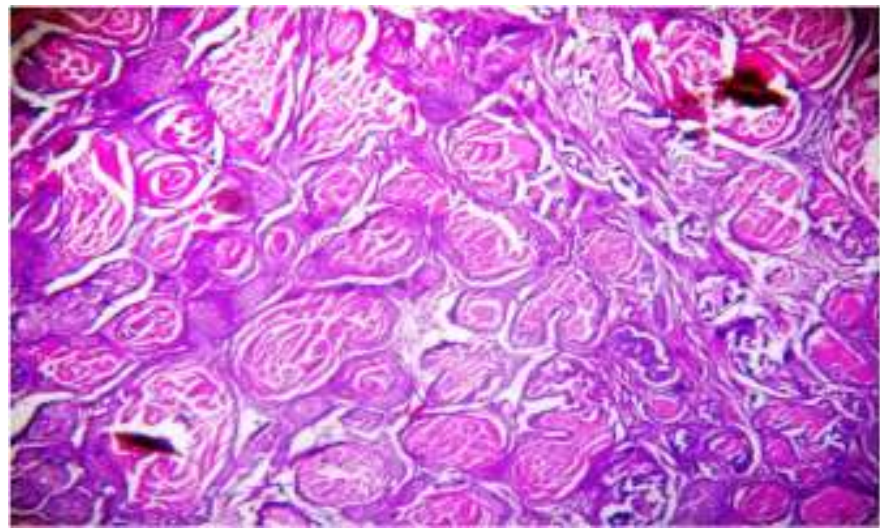

Fig.17: Trichoadenoma: Numerous horn cysts containing keratin and lined by eosinophilic cells

\section{Conclusion}

The skin appendageal tumors are uncommon and they constitute a minor percentage of the dermatology cases, reported by the pathologists. Many tumors have similar clinical presentation and diagnosis by the clinicians becomes difficult. The skin appendageal tumors exhibit characteristic histological pattern and features unique and helpful to identify them. Hence histopathology plays a major role and is essential and definitive for their diagnosis. They have predilection for face and scalp. Most of the skin appendageal tumors have a benign behavior and surgical excision is curative. This study is a sincere attempt for familiarity with the features of skin appendageal tumors and aid in their diagnosis.

\section{References}

[1]. Nair PS . A clinicopathologic study of skin appendageal tumors.Indian J Dermatol Venereol Leprol. 2008 Sep-O[6] ct; 74(5):550.

[2]. Gayathri SS, Ezhilvizhi A, Ashok kumar S. An analysis of skin appendageal tumours in South India. J of evol and den sci. 2012;1:907-12.

[3]. Samaila M. Adnexal skin tumors in Zaria, Nigeria. Ann Afr Med [serial online] 2008 [cited 2012 Sep 21]; 7:6-10.

[4]. Yaqoob N, Ahmad Z, Muzaffar S. Spectrum of cutaneous appendage tumors at Aga Khan University Hospita . J Pak Med Assoc. 2003 Sep; 53(9):427-31.

[5]. Rajalakshmi. V, Satish selvakumar, Rajeswari.K, Case series of skin adnexal tumors, Journal of clinical and diagnostics research, 2014,Sep.Vol-8(9) FC07-FC-10

[6]. Elder D, Elinistas R, Ragsdale BD. Tumours of the epidermal appendages. In:Elder D, Elinistas R,Jaworsky C, Johnson B Jr , editors. Levers Histopathology of the skin .8 th ed. Philadelphia:Lippincott Williams and Wilkins ;1997.p.747- 803.

[7]. Obaidat NA, Alsaad KO, Ghazarian D. Skin adnexal neoplasms-part 1 :An approach to tumours of the pilosebaceous unit. J Clin Pathol. 2007;60:129-44.

[8]. Obaidat NA, Alsaad KO, Ghazarian D.Skin adnexal neoplasm-part 2: An approach to tumours of cutaneous sweatglands. J Clin Pathol. 2007;60:145- 59.

[9]. Jayalakshmi, Looi LMi P. Cutaneous adnexal neoplasms in biopsy specimens processed in the Department of Pathology, University of Malaya. Ann Acad Med Singapore1996 July; 25(4):522-5.

[10]. Eleonora Giorgini1*, Gregorio Tugnoli1,Malignant Nodular Hidradenocarcinoma Arising on the Areola of a Male, J.Nucl.Med Radiat Therap,2012.S-2003

[11]. Kaleeswaran AV, Janaki VR, Sentamilselvi G, Kiruba MC. Eccrine spiradenoma. Indian J Dermatol Venereol Leprol 2002:68:236-

[12]. Ken Hashimotos, Tumors of Skin Appendages in Fitzpatrick Dermatology in General Medicine, Fitzpatrick TB 5 th edn Mc Grawhill 1999; 890-914.

[13]. Usha Ku Burra MD, Avninder Singh MD, Sunita Saxena MD DCP,Eccrine porocarcinoma (malignant eccrine poroma): A case report Dermatology Online Journal 11 (2): 17

[14]. K.Radhika, B. V. Phaneendra, N. Rukmangadha, and M. K.Reddy, "A study of biopsy confirmed skin adnexal tumors: experienceat a tertiary care teaching hospital,” Journal of Clinicaland Scientific Research, vol. 2, pp. 132-138, 2013.

[15]. U.Jindal, R. Patel : Study Of Adnexal Tumors Of The Skin: A Three Year Study Of 25 Cases. The Internet Journal of Pathology. 2012 Volume 13 Number 3. DOI: 10.5580/2bf5[PUBMED]

[16]. Julian CG. Bowers PW. A clinical review of 209 pilomatricomas. J Am Acad Dermatol 1998;39:191-5. .

[17]. Ankit Sharma, Deepak G. Paricharak, Jitendra Singh Nigam Histopathological Study of Skin Adnexal tumors- Institutional study in south India ,Journal of Skin Cancer,Volume 2014,

[18]. A. Saha, N. K. Das, R. C. Gharami, S. N. Chowdhury, and P. K.Datta, "A clinico-histopathological study of appendageal skintumors, affecting head and neck region in patients attendingthe dermatology opd of a tertiary care centre in eastern india,'Indian Journal of Dermatology, vol. 56, pp. 33-36, 2011 